\title{
Pavlovian conditioning to contexts that precede the place where shock occurs as measured by freezing and activity suppression in mice
}

\author{
LYNN J. HAMMOND \\ Temple University, Philadelphia, Pennsylvania
}

\begin{abstract}
Three experiments were conducted to demonstrate that the place where an organism has been, before the organism is moved to a place with aversive consequences, can also become aversive through classical conditioning. In Experiment 1, two groups of 8 mice were exposed to three different contexts in succession, with a single shock occurring in the third context. The distal context was a putative 3-min conditioned stimulus (CS) for freezing; the second context was a delay manipulation; and the unconditioned stimulus (US) occurred in the proximal context. The group delayed for 15 sec showed significantly more freezing to the distal CS context than did the group delayed for $3 \mathrm{~h}$. In a second experiment, conditioning to the distal context was demonstrated with a discrimination procedure for 8 more mice by using two different distal contexts as CS+ and CS - for the proximal context with shock. On $\mathrm{CS}+$ days, 3 min of exposure to the distal context was followed within $5 \mathrm{sec}$ by placement in the proximal box where shock occurred, whereas on CS- days, exposure to a second distal context was followed immediately by return to the home cage. Very strong differences in freezing between the $\mathrm{CS}+$ and CS- distal contexts were found in all 8 mice after 14 days of conditioning. In a third experiment, the discriminative procedure was repeated for 9 more mice, with two changes. More objective stabilometertype activity measures were substituted for observed freezing, and, in addition to the CS+ and CS - distal context trials, each mouse was also exposed to a third discriminative distal context, which was followed by $15 \mathrm{~min}$ in a delay chamber followed by shock in the proximal context. This discrimination procedure with the activity suppression measure again resulted in significant differences between the contexts. The CS+ context and the context followed by a 15-min delay did not differ, but both of them differed from the CS- context.
\end{abstract}

Under natural or experimental conditions wherein Pavlovian conditioning can occur, the organism is typically in different contexts before it is in the context in which the unconditioned stimulus (US) occurs. It is possible that the contextual cues of the antecedent place or places could become associated with the US. Because organisms typically move through complex environments, such possible conditioning to the antecedent contexts could play an important role in the elicitation and release of relevant behaviors. The literature on spatial learning, such as that on maze running with rats, contains numerous examples of how behavior is controlled by contextual cues distal from reinforcers at the goal; and this stimulus control could be operant, Pavlovian, or some combination of both.

This paper is concerned with the possibility that some Pavlovian conditioning can occur to antecedent contexts. In discrete stimulus conditioning, it has been repeatedly demonstrated (see, e.g., Kehoe, 1982) that conditioning to the more distal of serially presented conditioned stimuli (CSs) can take place. Also, it is well established that con-

Reprints can be obtained from the author, Department of Psychology, Temple University, Philadelphia, PA 19122 (e-mail: lhammond@) astro.ocis.temple.edu).

-Accepted by previous editor, Vincent M. LoLordo textual stimuli can readily be classically conditioned (see, e.g., Balsam \& Tomie, 1985), and the context conditioning literature contains findings that are similar to, but not the same as, what is referred to here as antecedent contextual conditioning. Marlin (1983) has reported that pairing a putative discrete CS with an aversive context produced Pavlovian conditioning (based on second-order conditioning); in addition, Helmstetter and Fanselow (1989) found that pairing a new context with an aversive CS also produced second-order conditioning. Given these findings, it would not be surprising to discover that a neutral context could become classically conditioned when quickly followed by USs occurring in a different context (antecedent contextual conditioning). Another relevant finding that appears to involve antecedent contextual conditioning but in fact does not comes from studies incorporating longdelay contextual conditioning procedures. For example, Williams, Frame, and LoLordo (1992) found that contextual stimuli become aversive even when the shock US is not presented until $20 \mathrm{~min}$ into the session, thus creating a 20-min delay between the onset of the contextual stimulus complex and the US. As conceptualized here, this would not be an instance of antecedent contextual conditioning, because the putative CS is actually present when the US occurs. This paper focuses on a procedure whereby the putative contextual CS precedes the US but is not present when 
the US occurs-a trace or serial procedure. This procedure does not appear to have been investigated previously.

For many, a successful demonstration of antecedent contextual conditioning would raise the issue of how such conditioning takes place. In the discrete CS literature on serial conditioning, it has been shown that the mechanisms mediating such distally occurring discrete CS conditioning are quite complicated and that there are several ways in which such distal CSs can gain associative strength. First, trace conditioning can take place between the distal CS and the US, which would be independent of the proximal $\mathrm{CS}$ in the CS series. Second, conditioning to the distal CS can be dependent on second-order conditioning to the proximal CS as a US. Third, conditioning can be based on an interaction between the distal and proximal CSs that is not based on trace or second-order conditioning or on stimulus generalization. Rescorla (1982) has shown that such a gapfilling effect of an intervening $\mathrm{CS}$ in serial conditioning does not have to be based on second-order conditioning.

However, in contextual serial CS paradigms such experiments cannot be designed as easily, because the procedure of contextual trace conditioning without a gap-filling context cannot be directly produced as it can with the discrete CSs. (Removal of one set of contextual stimuli requires placing the animal into a completely new set of contextual stimuli-a set that was not there previously.) Because of these complexities, investigation into the mechanism of antecedent contextual conditioning has been deferred to later studies. The purpose of this study was to conclusively demonstrate conditioning to contextual, distal CSs while controlling for such confounding effects as stimulus generalization between distal and proximal contexts and pseudoconditioning. Any or all of the three mechanisms discussed above could produce conditioning to the distal situation in this kind of contextual conditioning. In this study, we used mice and measured freezing behavior or the suppression of fine motor behavior in response to the conditioned contextual cues.

\section{EXPERIMENT 1}

An important characteristic of antecedent contextual conditioning, as defined above, would be that the putative CS be a different place from that in which the US occurs. In naturalistic settings, contexts change gradually as the organism locomotes through its environment, and what constitutes an individual place is somewhat open to judgment. Therefore, it was important that the distal contextual did not overlap the proximal context. One way to ensure this was to place the mouse in a third context in between the proximal and the distal CSs. This would constitute either a trace or a serial contextual conditioning procedure. In order to minimize the length of this trace or gap between the distal and the proximal contexts, the mice were mechanically lowered quickly through a stack of three chambers. This procedure ensured that the distal and proximal contexts were never in the mouse's view at the same time, yet the trace could be minimal. Pilot work with this three-chamber arrangement suggested that substantial freezing to the distal context could be observed even with trace intervals of more than a few seconds.

Consequently, antecedent contextual conditioning was examined in a simple two-group experiment in which the proximal CS did not occur immediately after the distal CS. The experiment controlled for possible aversive consequences of lowering the mice from one chamber to another. The two groups differed in the length of time spent in a delay chamber, and thus also differed in the temporal interval between the distal CS and the proximal CS. The delay context treatment was divided into an early delay and a late delay period, with a fourth chamber added for the early delay period. Both groups were lowered into the early delay chamber and then, either immediately $(15-\mathrm{sec}$ group) or after $3 \mathrm{~h}$ (3-h group), they were placed by hand into the late delay chamber and lowered into the proximal CS chamber where the US occurred.

\section{Method}

Subjects. Sixteen female, Swiss-Webster outbred mice, approximately 6 months old, were obtained from a stock bred at Temple University and divided equally into a 15 -sec delay and a 3-h delay group. The mice were housed in rectangular stainless steel home cages $(24 \times 12 \times 12 \mathrm{~cm})$ with a wire mesh top and sawdust on the floor of the cage ( 4 mice/cage).

Apparatus. In addition to the home cage, four other distinct experimental contexts or chambers were used. The distal CS chamber was a $20 \times 15 \times 16 \mathrm{~cm}$ wooden box with a clear plastic removable top and a floor composed of two equal-sized, smooth metal rectangles, which divided the floor exactly in half, with the separation in the long dimension of the box. All wood and metal interior surfaces were painted with glossy white enamel. The two parts of the floor were hinged so that the mice could be lowered. A small mirror was mounted above the clear plastic top so that the mice could be observed. The proximal CS chamber contained twenty 2-mm stainless steel shock grids and measured $23 \times 20.5 \times 19 \mathrm{~cm}$. The front and back were clear plastic, and the two sides were unpainted metal. The grids in the proximal CS chamber received scrambled current from a Grason-Stadler shock generator, Model 700. The early delay chamber consisted of a number of light gray plastic, open-topped commercial housing cages $30 \times 30 \times 10 \mathrm{~cm}$ in size, which had approximately $3 \mathrm{~cm}$ of sawdust on the floor. Two wooden rails were placed on top of this early delay chamber to hold the distal CS chamber and allow its hinged floor halves to swing down. The rails and the hinged floor of the distal CS chamber formed the ceiling of the early delay chamber, and a string and pulley system allowed the experimenter to gently lower the mouse from the distal CS chamber to the early delay chamber by releasing the hinged floor of the distal CS chamber. The distal CS chamber and the rails could be quickly lifted off the early delay chamber and replaced by a commercial stainless bar top. In this way, many mice could be stored in these early delay chambers while the next mouse received the beginning of a trial. The late delay chamber, $20.5 \times 17 \times 15 \mathrm{~cm}$ in size, had a hinged floor similar to that of the distal CS chamber, and the top, sides, and floor surfaces were covered with fine (No. 600) dark gray sandpaper. When confined in the late delay chamber, the mouse could not see out of the chamber except through the cracks in the drop floors. The late delay chamber was attached to the top of the proximal CS chamber, and the mouse could be lowered into the proximal CS chamber through the hinged floor in a manner similar to that in which transfer occurred from the distal CS chamber to the early delay chamber. White noise of approximately $85 \mathrm{~dB}$ was always produced by a speaker $1 \mathrm{~m}$ above the experimental chambers throughout the experimental sessions.

Procedure. During the 1 st week, the mice were given a 15 -sec handling pretreatment on 4 separate days to tame them and reduce 
the likelihood of freezing from handling alone. Subsequently, on each day of regular experimental treatment, the home cages of the mice were placed on a cart and wheeled from the colony to the laboratory on the floor below. The cart with all the mice in the experiment was placed in an anteroom to the laboratory room. For all mice, a trial involved removing the mouse by hand from its shared home cage and carrying it the four or five steps to the apparatus in the adjoining room.

The mouse was placed in the distal CS chamber, and the lid was replaced. The mouse remained in the distal CS chamber for $3 \mathrm{~min}$, at the end of which it was gently lowered into the early delay chamber and the distal CS chamber and the rails were removed. For the 3-h delay group, the lid was attached to the early delay chamber and it was set aside for $3 \mathrm{~h}$. Afterward the mouse was taken from the early delay chamber and placed (by hand) into the late delay chamber. The mice in the 15-sec delay group were immediately lifted out of the early delay chamber after being lowered into it and then placed by hand into the late delay chamber. Once each mouse in both groups was in the late delay chamber, it remained there for $10 \mathrm{sec}$ and was then lowered into the proximal CS chamber. There it received a 2 -sec, 1.6-ma shock $3 \mathrm{sec}$ after entry into the proximal CS chamber. Each mouse always remained in the proximal CS chamber for $1 \mathrm{~min}$ before it was returned to the home cage.

For the 15-sec delay group, the time from leaving the distal CS chamber until entering the proximal CS chamber was approximately $15 \mathrm{sec}$. For the 3-h delay group, this period was approximately $3 \mathrm{~h}$. There were 2 conditioning days, with one trial per day, and a 3rd extinction test day during which the mice were placed one at a time into the distal CS chamber and their freezing in that chamber was scored for $3 \mathrm{~min}$. Freezing was measured according to the standard time sampling technique (see, e.g., Fanselow, 1990). In this case, behavior was sampled once every $5 \mathrm{sec}$ during the stated observation periods, and the mouse was scored as freezing or not freezing in each instance. The observation was made at exactly the 5-sec mark. If the mouse remained motionless (except for breathing movements) for approximately I sec, it met the freezing criterion. If any movement other than breathing occurred during that 1 sec, it was scored as nonfreezing.

To summarize - all mice were exposed to all four of the experimental contexts in the same sequence (distal CS, early delay, late delay, and proximal CS). Each mouse received the same amount of handling and lowering from one chamber to the next, including the home cage. Only the length of time that they spent in the early delay box varied.

\section{Results}

The total amount of freezing differed between the two groups throughout the 3-min test period. The 15-sec delay group mice froze a mean of $52 \%$ of the time, whereas the 3-h delay group mice froze a mean of $17 \%$ of the time. Almost all the freezing in the long delay group occurred in the $1 \mathrm{st}$ min. This pattern of freezing is shown in Figure 1. A two-way analysis of variance (ANOVA) with one repeated factor (minutes) was conducted for this pattern: both the groups and the minutes factors were significant, but the interaction was not $[F(1,14)=14.21, p<.01 ; F(2,24)=$ $15.6, p<.01$, respectively].

\section{Discussion}

Because the two groups were treated in an almost identical fashion, except for the amount of time spent in the early delay chamber, the large difference in freezing between them must have been produced by the delay factor and not some other effect, such as the possible aversive consequences of falling, or generalization based on some

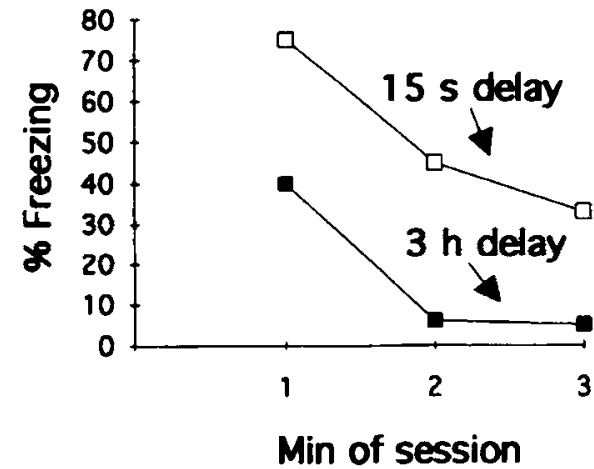

Figure 1. Mean percent freezing for the two groups of Experiment 1 , plotted for the 3 -min test period as 3 separate minutes.

common cue between distal and proximal chambers for one group, but not the other. The difference in freezing must be a Pavlovian conditioned response based on the temporal relation between the distal CS chamber and the proximal CS chamber or its shock. Thus, it can be concluded that one context can be reinforced by the events occurring in a substantially different context. It can also be seen that at least two separate contexts and $15 \mathrm{sec}$ of time can intervene between the distal and the proximal CS context, yet conditioning can still occur to the distal CS. Finally, note that the 3-h delay group did show rather noticeable freezing (40\%) during the 1 st min of the distal CS test period (even though it was only about one half as much freezing as in the short delay group).

\section{EXPERIMENT 2}

The results of Experiment 1 indicate that a fairly brief interval between one context and a second context in which a US occurs will produce substantial conditioning to the first context. But it was also found that some noticeable amounts of freezing occurred even when the interval was quite long. The latter effect could be due to substantial generalization from the shock chamber to the test chamber. If such generalization is the result of some common cues between the distal and proximal chambers, and if these cues are common to most of our laboratory chambers (in spite of the cue differences we observe), then the use of a discrimination procedure for the distal CS stage could substantially reduce this generalization by extinguishing the common cue elements each time a partially different, distal CS - chamber was not followed by shock during the unreinforced discrimination trials.

In Experiment 2, such a discrimination was examined. In addition to reducing generalization from proximal to distal CSs, differential conditioning could also reduce generalization based on other common cues, such as handling when animals were placed in the distal and proximal CS contexts. Therefore, in this experiment, a more typical procedure of animal handling was used. It did not include lowering the mouse, for the reason given above and because Experiment 1 demonstrated that conditioning would 
occur even with substantial intervening events, including handling, occurring between the distal and proximal contexts. The discriminative conditioning was conducted with two different distal contexts as CSs: one that preceded the proximal $\mathrm{CS}$ and shock by seconds (CS+) and one that preceded it by a day ( $\mathrm{CS}-$ ).

\section{Method}

Subjects. Eight naive mice from the same source as above were used as subjects, and they were housed and maintained in the same fashion as in the previous experiment.

Apparatus. In addition to the distal and proximal CS chambers from the previous experiments, a third, distal CS chamber was used that consisted of a round, black, soft-textured chamber, $20 \mathrm{~cm}$ in diameter, $20 \mathrm{~cm}$ high, with the same size of floor area as that of the square, white, distal CS chamber. This round, black chamber had heavy black felt fabric on the interior walls; the floor was black terrycloth; and the ceiling was a flat board painted black, with a small 4W bulb attached to the center of the ceiling. A small $8 \times 8 \mathrm{~cm}$ oneway mirror was set into the side of the cylindrical chamber, so that when the room was darkened, the experimenter could see the mouse, but the mouse could not see the experimenter. As a further set of discriminative cues, for the square, smooth, white distal box, both the mouse and the experimenter could see each other through the mirror above the chamber ceiling as before, and the mouse could see into the room; but none of those "extra apparatus" cues were available when the mouse was in the round chamber. In these ways, the two distal CS chambers were constructed to be as different as possible. All three chambers were placed side by side on a table with the proximal box exactly halfway between the two distal chambers.

Procedure. This single group of mice was pretreated and transported in the same manner as in the previous experiment. The daily procedures alternated between a CS+ trial on one day and a CStrial on the next. On the CS+ trials (odd-numbered days) each mouse was taken by hand into the experimental room and placed in the appropriate distal CS chamber. At the end of $3 \mathrm{~min}$, the mouse was removed from the top of the distal chamber and quickly placed by hand through the front door into the proximal chamber. The experimenter then quickly crossed the room and changed the room lights (from dark to light if the mouse had been in the round chamber, from light to dark if the mouse had been in the square chamber), and a 2-sec, 1.6-mA shock was delivered $1 \mathrm{sec}$ later. The light change was done to reduce the possibility that the lighted or darkened condition of the room could serve as a common cue for generalization between the distal chamber and the proximal chamber on $\mathrm{CS}+$ trials. The changing of the lights meant that the lighting conditions during the distal box portion of the CS+ trial would be the opposite cue from that present during shock. Thus if the lighting change functioned as a cue for shock, its opposite condition would be present during the measurement of freezing in the CS+ distal chambers but not the CS - distal chambers (i.e., there was conservative biasing against the desired experimental outcome). The entire procedure from removal from the distal CS chamber to shock onset in the proximal chamber took approximately $5 \mathrm{sec}$. The mouse remained in the proximal chamber for $1 \mathrm{~min}$ and then was removed and returned to a holding cage in the adjoining room. Four mice received the round distal chamber as a $\mathrm{CS}+$, and the other 4 received the square distal chamber as the CS+.

On even-numbered or $\mathrm{CS}-$ days, the procedure of placement in the distal chamber was repeated but the opposite chamber was used, so that a mouse that had the round chamber on $\mathrm{CS}+$ days would have the square one for $\mathrm{CS}-$ days, and so forth. Also, when the animal was removed from the distal CS chamber at the end of $3 \mathrm{~min}$, it was taken directly out of the experimental room to the holding cage in the adjoining room. Four mice received the round distal chamber as a $\mathrm{CS}-$ in the darkened room, and the other 4 mice received the square distal chamber as the CS - in the lighted room. The differential conditioning procedure was repeated for 14 consecutive days, so that there were seven CS+ trials and seven CS - trials. Freezing was scored in the same manner as before each time a mouse was in either a CS+ or a CS - distal chamber on every trial.

\section{Results and Discussion}

Figure 2 shows mean freezing data for the 4 mice that received the square chamber as $\mathrm{CS}+$ and round one as CS - and means for the 4 mice that received the opposite stimuli for CS+ and CS - across successive distal stimulus exposures. Data from the first trial (a CS+ one for all mice) reflect the initial effects of the chamber itself, not its association with shock, since shock had not yet occurred. For the 4 mice in the square chamber, no freezing was observed (as is typically found for most chambers in our laboratory in other studies where no-shock control mice have been used). But for the round chamber there was a substantial amount of freezing $(14 \%)$ on the pretest trial. Figure 2 also reveals that with both counterbalanced sets of contexts, very substantial discriminative conditioning was present by the end of the conditioning procedure. However, as can readily be seen, this discrimination was much more rapid for the mice receiving the round chamber as a CS+, than for the counterbalanced mice with the round chamber as CS - . Perhaps the round chamber was somewhat aversive even before shock occurred because some residual pheromone remained in the fabric from mice for whom this chamber had been a CS $+24 \mathrm{~h}$ earlier and this accounted for the slower acquisition of the discrimination in the mice with the square as CS+.

Nevertheless, all mice eventually developed very substantial discriminations, no matter what chamber served as $\mathrm{CS}+$ or $\mathrm{CS}-$. The discriminations, as reflected in the test scores on the last 2 days for individual mice, are shown in Figure 3, and the overall difference between CS + and CS- was significant (Wilcoxon $T=0, n=8, p<.01$ ).

The apparent aversiveness of the round chamber introduced a conservative bias for the demonstration of a discrimination when that fearful stimulus served as the CSfor half of the mice in this experiment. The fact that not being shocked following exposure to this chamber even-

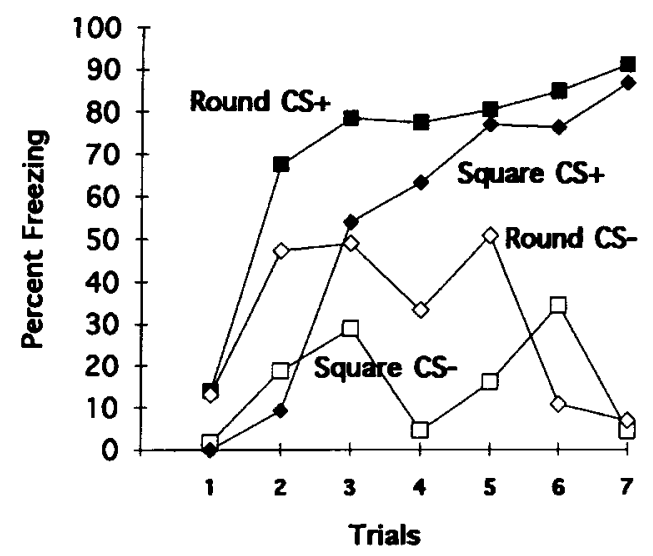

Figure 2. Mean discriminative freezing for the half of the subjects in Experiment 2 which had the round context as CS+ and the other half which had the square context as CS+. 


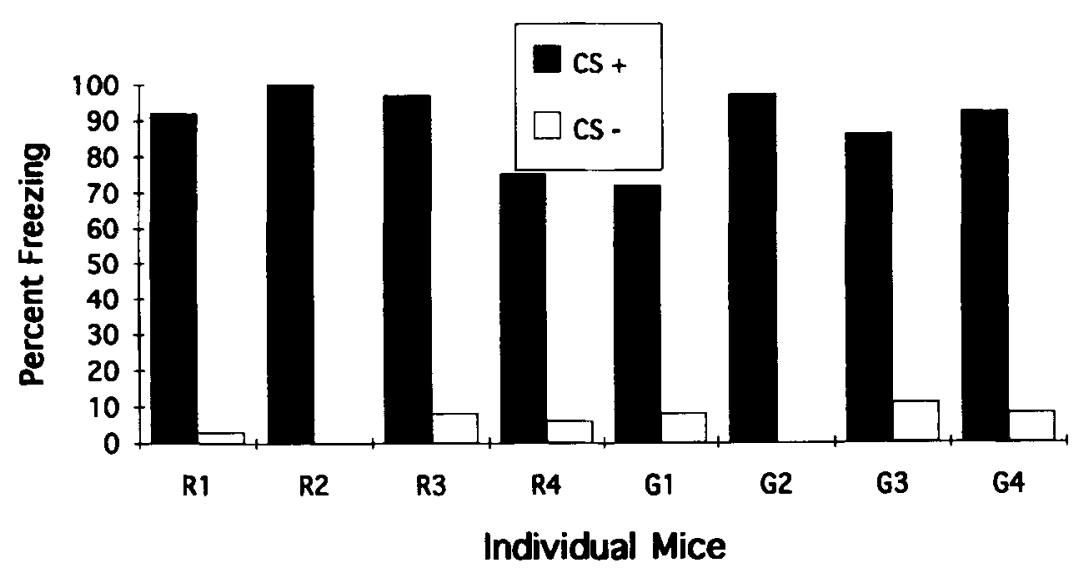

Figure 3. The final discrimination for each of the 8 mice in Experiment 2. Each CS+ trial is from Day 13 and each CS - trial is from Day 14.

tually overcame the initial aversiveness of that chamber argues strongly for the interpretation that it was the very short delay between the distal CS+ chamber and the proximal CS/US chamber that led to the development of a dominating amount of freezing, whereas the very long delay between the distal CS - chamber and the proximal CS chamber with shock eventually led to very little freezing.

\section{EXPERIMENT 3}

As previously stated, the purpose of this study was to explore the possibility that respondent behavior can play a role in determining how an organism interacts with its spatial environment. Such an influence of Pavlovian conditioning could follow from the conditioning of contextual stimuli that precede those where the US occurs, and such conditioning was demonstrated in Experiments 1 and 2. How important a role this conditioning would play in typical spatial situations would also depend on the temporal gradient between the distal and proximal contexts in such antecedent contextual conditioning. With longer intervals between them, less and less conditioning should occur, until an interval is reached which produces no conditioning at all. In Experiment 1, a 15-sec delay between the distal and proximal serial CSs produced substantial conditioning to the distal stimulus.

The purpose of Experiment 3 was to investigate a longer delay of $15 \mathrm{~min}$. Given the strong antecedent contextual conditioning seen with the discrimination procedure, this discriminative approach was extended to investigation of a much longer delay. The mice in this experiment were exposed to a three-stimulus discrimination, in which three different distal contexts were used for each mouse. Each day the mouse was exposed to one of these three contexts and then placed in the proximal CS chamber immediately and shocked (CS+ condition), or placed in a delay chamber for $15 \mathrm{~min}$ before it was placed in the proximal CS box and shocked (CS delay + condition), or returned to its home cage until the next day (CS- condition). Every 3 days each mouse was exposed to all three of these conditions.
Another goal of Experiment 3 was to use a nonobservational conditioning measure. The freezing data of the first two experiments were based entirely on the observations of a single experimenter (in part because the nature of the contextual stimuli in Experiment 2 precluded video recording of the sessions). In order to increase the objectivity of these observations, an automatically recorded, fine-motor activity measure was substituted for the freezing measure. Whenever freezing takes place in a chamber, activity should be lowered overall. Bouton and Bolles (1980) found a strong correlation between freezing and conditioned suppression of operant behavior in rats. They also demonstrated suppression of untrained activity overall in rats in their third experiment, and a similar procedure with rats was used by Kraemer, Randall, and Carbary (1991). Finally, Kameyame and Nagasaka (1982) have used activity measures to index conditioned suppression in mice that had not received any operant training. This contextshock-produced suppression of unlearned behavior in mice was used as the measure of conditioning in the experiment below. If the mice were to develop low levels of activity in the CS+ context, intermediate levels in the CS delay+ context, and high levels of activity in the CS - context, then differential conditioning to the three different distal CSs would be demonstrated.

\section{Method}

Subjects. Nine naive mice from the same source as above were used as subjects, and they were housed and maintained in the same fashion as in the previous experiments. There were six combinations of the three physical contexts and the three types of US consequence $(+,-$, and delay). Two mice were assigned to each of three combinations, and one mouse to each of the remaining three.

Apparatus. The proximal CS shock chamber, the square, white, smooth chamber, and the round, black, fabric-lined distal CS chambers from Experiment 2 were used in the same fashion in this experiment. A third distal CS chamber was added, which was of intermediate brightness (tan cardboard), rectangular in shape $(31 \times 9 \times$ $16 \mathrm{~cm}$ ), with a floor made of metal window screening resting directly on a piece of plywood. Each chamber had a removable top of clear plastic. In addition, the walls of each of the three distal chambers did not rest directly on the floor material (metal, fabric, or screen) 
but were raised several millimeters so that the walls and ceiling floated over the floor and the wooden platform on which the floor material was resting. This was done because the wooden platform was mounted on springs and measured activity levels through the use of a magnet mounted beneath the center of the platform. The magnet moved inside a stationary coil, which in turn generated a current that was fed into a Lafayette Instruments animal activity monitoring device, Model 86010 . Movement of the sprung platform generated relay closures in the monitoring device, which were then recorded on mechanical counters. The walls and ceiling were mounted just above the platform in each chamber, because their weights affected the sensitivity of the platform movement. Finally, the CS delay+ condition utilized a fifth type of chamber for the delay period (between the distal CS delay+ chamber and the proximal CS chamber), which consisted of a wooden box with a metal floor $(20 \times 17 \times 16 \mathrm{~cm})$ covered with a very rough textured paint that created a "salt and pepper" pattern of two shades of gray. In this experiment, the room lights were always on during all the procedures.

Procedure. Each mouse was exposed to the same handling pretreatments as in the previous experiments, and then they were given discrimination training which consisted of a single trial each day in which they were placed by hand into one of the distal chambers for $3 \mathrm{~min}$ and then removed by hand. On CS+ trial days, they were immediately placed into the proximal chamber, where they received a single 1.6-ma, 2.0-sec shock within $5 \mathrm{sec}$ of placement. They remained in the proximal CS chamber for $1 \mathrm{~min}$ and were then placed into a holding cage (plastic waste basket with sawdust on the bottom) until all 4 cagemates had been run, and then all 4 were placed in their home cages. On CS - trial days, they were placed immediately into the holding cage and then their home cage. On CS delay+ trial days, they were placed into the delay chamber for $15 \mathrm{~min}$ and then placed into the proximal CS chamber and given the same treatment as on the CS+ trial. All placements were done by hand in this experiment.

Each type of distal CS chamber (square, round, rectangular) served as CS+, CS-, and CS delay for 3 mice. That is, counterbalancing ensured that differences in CS effectiveness would not confound the effects of trial consequence. Every 3 days, each mouse received 1 day of each type of trial treatment, but the sequence of kind of treatment did not repeat in a regular fashion. This period where all conditions are each presented once is sometimes called a discrimination cycle. There were 39 days of discrimination treatment or 13 discrimination cycles. The activity measures taken in the distal CS chambers consisted of the total number of relay closures that occurred during the 3-min CS. These numbers ranged from 0 to over 1,000 .

\section{Results and Discussion}

Discrimination cycles are shown in Figure 4. (The first cycle is omitted, since for some subjects the stimulus presentation was a pretest but for other presentations shock had already occurred.) As can be seen, discrimination developed slowly and emerged by the sixth discrimination cycle. From the sixth cycle on, the least activity was seen to $\mathrm{CS}+$ and the most to CS-, with an intermediate level of activity to the delayed CS. The mean number of activity counts for all 9 subjects over the last five discrimination cycles was 200 for CS,+ 325 for CS delay+, and 494 for the CS-. The mean difference between CS+ and CStrials was found to be significant using a Wilcoxon, matched pairs, signed ranks test $(T=0, n=9, p<.01)$, and between CS delay+ and CS - trials $(T=0, n=9, p<$ $.01)$. The difference between CS+ and CS delay+ approached but did not reach significance $(T=7, n=9, p>$ .05 ), as a $T \leq 6$ was required for a two-tailed test. These mean discriminative differences were quite representative of the discriminations of individual subjects, which are

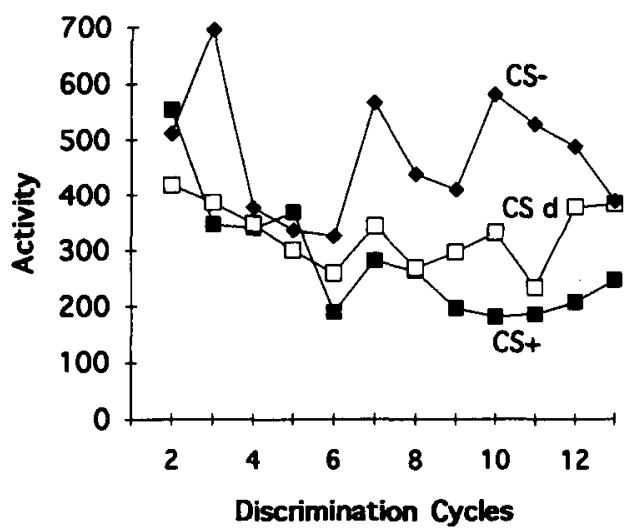

Figure 4. Mean activity levels for all mice for successive cycles of the three-stimulus discrimination in Experiment 3, beginning with the second cycle.

plotted as means for the last five discrimination cycles for each mouse in Figure 5.

The mechanical activity measure is totally objective, and the fact that all 9 mice showed greater activity to CSthan to CS+ confirms the findings based on observations of freezing from the preceding experiments that distal contextual CSs can become conditioned to shock occurring in the proximal contextual CS. Second, the fact that all 9 mice also showed more activity to the CS - than to the CS delay+ demonstrates that this type of antecedent contextual conditioning to the distal CS can occur with a temporal delay of as long as $15 \mathrm{~min}$ between the distal and proximal CS. The fact that the difference between the CS+ and the CS delay+ was not significant detracts in no way from these conclusions; in fact, it suppports the argument that no measurable conditioning was lost through the introduction of a 15-min delay between the distal and proximal CS.

\section{GENERAL DISCUSSION}

In discrete stimulus conditioning, the interval between the onset of the CS and the onset of the US (the interstimulus interval, ISI) is extremely important in determining the presence and amount of conditioning. Also, even very small gaps between the offset of the CS and the onset of the US can markedly attenuate conditioning (trace conditioning; see, e.g., Kamin, 1965). But in many recent investigations of contextual or situational conditioning, a number of USs have been presented during each context exposure. The effectiveness of these context procedures could suggest that ISI and trace variables are less descriptive, and that the number of times when there is contiguity between the presence of the CS and the US might be the more descriptive variable accounting for contextual conditioning. This would increase the distinction between contextual and discrete stimulus conditioning and in that way might also be at least a partial basis for the vague notion of "place conditioning" (see, e.g., Nadel \& Willner, 1980). 


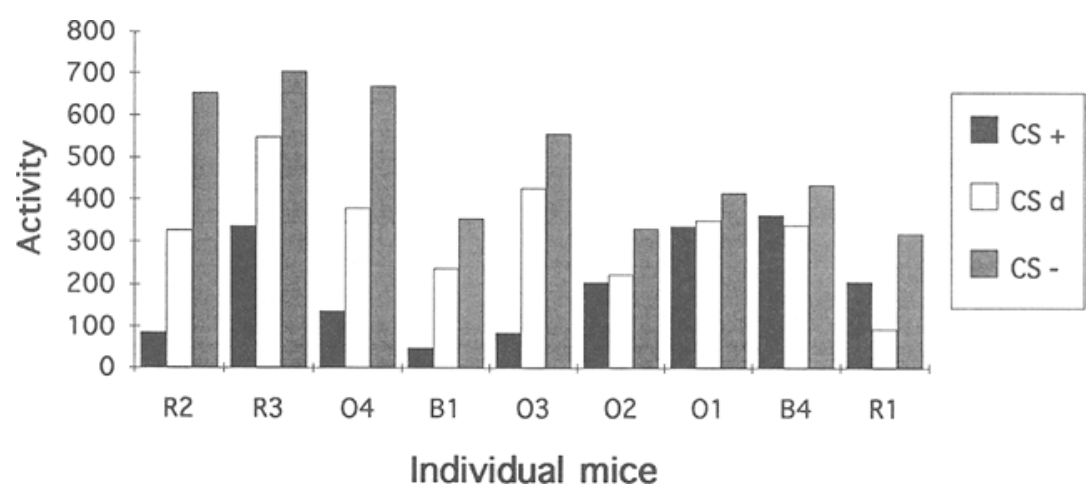

Figure 5. Mean activity levels for individual mice for the last five cycles of the threestimulus discrimination in Experiment 3.

In contrast to that argument, the paradigm introduced in this paper, antecedent contextual conditioning, manipulates contextual CSs and the US in a manner more similar to discrete stimulus trace or serial conditioning. The successful demonstrations of such contextual conditioning reported here further support the general argument that there is nothing unusual about contextual conditioning with regard to the ISI and trace variables. But perhaps of much more importance, the paradigm can be used to further explore the similarities and differences; if any, between contextual and discrete stimulus conditioning.

Future research in this area should also address the issue of what mechanisms underlie conditioning to the distal $\mathrm{CS}$, since the present study does not provide information about these possible mechanisms. As noted before, three explanations and procedures for their evaluation have been reported in the fairly recent discrete CS literature, but complex problems arise when similar analyses and procedures are conceptualized for purely contextual conditioning. To turn to the first explanation, the proximal CS may be unnecessary and the conditioning to the distal CS may be produced by its trace relation to the US. In the relevant discrete CS literature, this has been evaluated through comparison with a condition or group for which the proximal CS has been omitted. Frequently, it has been shown that presenting a proximal $\mathrm{CS}$ during the trace interval produces a sizable and significant increase in conditioning to the distal CS (see, e.g., Rescorla, 1982). Although it is possible to present no discrete CS for some period of time, it is not possible to have the absence of any contextual CS for some period of time, because the organism is always exposed to some set of contextual stimuli. In that sense, simple trace conditioning procedures cannot be implemented with strictly contextual conditioning paradigms. The comparison between conditioning in which no $\mathrm{CS}$ intervenes between the distal CS and the occurrence of the US and the condition in which such a putative gapfilling CS is present has been critical to the current analysis of the mechanisms of distal CS conditioning. If no difference is found in this comparison, one can argue that the proximal CS has no effect and conditioning is of the simple trace variety. If a difference is found, this sets the stage for analysis of the other two mechanisms described below, second-order conditioning or a "catalytic" gap-filling effect. In other words, this comparison has been necessary for analysis of each of the three possible mechanisms advanced in the discrete CS literature. Therefore, it will be necessary to develop a new method of analysis in order to answer the question of whether the conditioning observed to the distal contextual CSs is produced by simple trace conditioning or is the result of some interaction with one or more proximal CSs.

As just mentioned, the second explanation of distal CS conditioning is based on second-order conditioning. The proximal CS becomes conditioned, and then it functions as a second-order US to condition the distal CS with which it is always contiguous. Rescorla (1982) found conditioning to discrete, distal CSs that was not produced by secondorder conditioning. This conclusion was again based on comparisons with a condition in which there was no gapfilling stimulus (i.e., trace conditioning). As previously noted, such a comparison cannot be made with contextual gap-filling stimuli. It might be thought that one could simply extinguish the proximal contextual CS after antecedent contextual conditioning has taken place in order to demonstrate whether or not second-order conditioning is playing a role (Rescorla, 1982, Experiment 3). But if this results in no effect, as it did in Rescorla's case, one does not know whether that null outcome is the result of a lack of secondorder conditioning or was produced by mediation of the second-order conditioning by a stimulus-response mechanism. Consequently, in order to rule out second-order conditioning, Rescorla (1982, Experiment 4) returned to the comparison procedure between gap-filling proximal stimulus and no gap-filling proximal stimulus in order to draw conclusions about the role of second-order conditioning in producing conditioning to the distal CS.

Negative findings in the discrete CS literature for the two explanations above (trace and second-order conditioning) have led by default to the third kind of explanation, which is less an explanation than a demonstration that there is an interaction between the two CSs that is not based on second-order conditioning. Thus, once again, as with the first two explanations, this third one has also been depen- 
dent on the critical comparison between proximal CS and no proximal CS conditions which cannot be made for contextual CSs. In the present discussion, it is not intended to argue that the mechanism of distal context conditioning cannot be analyzed, only that it will require new methods.

A practical consequence of the successful demonstration of sizable conditioning to an antecedent context is that investigators of Pavlovian conditioning should now be more attuned to the possible confounds that could arise in their experiments from the conditioning of cues that occur before the session as defined by them. Cues such as room characteristics, experimenter characteristics, or the nature and kind of handling that may often be taken for granted could become Pavlovian discriminative cues for what is to follow in that particular session.

Another implication, of a more general theoretical nature, is that researchers interested in how the organism learns about its spatial environment can now be a little more likely to entertain a possible role for Pavlovian conditioning mechanisms in such spatial learning. Given the demonstrations here, it would appear that the organism is capable of acquiring Pavlovian conditioned incentive properties to serial sequences of spatial contexts as it moves through its environment. This is, of course, a very old ideathe cornerstone of incentive motivation theory (see, e.g., Bindra, 1974; Mowrer, 1960). Perhaps this particular set of procedures could be used in the service of current investigations of incentive motivation theory.

\section{REFERENCES}

Balsam, P. D., \& Tomie, A. (Eds.) (1985). Context and learning. Hillsdale, NJ: Erlbaum.
BINDRA, D. (1974). A motivational view of learning, performance and behavior modification. Psychological Review, 81, 199-213.

Bouton, M. E., \& Bolles, R. C. (1980). Conditioned fear assessed by freezing and by the suppression of three different baselines. Animal Learning \& Behavior, 8, 429-434.

FANSELOW, M. S. (1990). Factors governing one-trial contextual conditioning. Animal Learning \& Behavior, 18, 264-270.

Helmstetter, F. J., \& Fanselow, M. S. (1989). Differential secondorder aversive conditioning using contextual stimuli. Animal Learning \& Behavior, 17, 205-212.

KAMEYAME, T., \& NAGASAKa, M. (1982). The effects of analgesics on quickly-learned conditioned suppression in mice. Neuropharmacology, 21, 1283-1289.

Kamin, L. J. (1965). Temporal and intensity characteristics of the conditioned stimulus. In W. F. Prokasy (Ed.), Classical conditioning (pp. 118-147). New York: Appleton-Century-Crofts.

KEHOE, E. J. (1982). Conditioning with serial compound stimuli: Theoretical and empirical issues. Experimental Animal Behaviour, 1, 30-65.

Kraemer, P. J., Randall, C. K., \& Carbary, T. J. (1991). Release from latent inhibition with delayed testing. Animal Learning \& Behavior, 19, 139-145.

MarLIN, N. A. (1983). Second-order conditioning using a contextual stimulus as $\mathrm{S}_{1}$. Animal Learning \& Behavior, 11, 290-294.

MOWRER, O. H. (1960). Learning theory and behavior. New York: Wiley.

NADEL, L., \& Willner, J. (1980). Context and conditioning: A place for space. Physiological Psychology, 8, 218-228.

RESCORLA, R. A. (1982). Effect of a stimulus intervening between CS and reinforcer in autoshaping. Journal of Experimental Psychology: Animal Behavior Processes, 8, 131-141.

Williams, D. A., Frame, K. A., \& LoLordo, V. M. (1992). Discrete signals for the unconditioned stimulus fail to overshadow contextual or temporal conditioning. Journal of Experimental Psychology: Animal Behavior Processes, 18, 41-55.

(Manuscript received August 17, 1992; revision accepted for publication September 20, 1994.) 